\title{
Educación universitaria y ciudadanía global: ¿puede la igualdad de género ser optativa?
}

\author{
Ana Iglesias Galdo \\ Universidade da Coruña | Espanha
}

\section{Resumen}

Reclamando las contribuciones de los estudios de mujeres, feministas o de género, enfocaremos el ámbito universitario bajo dos propósitos: evidenciar algunos datos que, a modo de radiografías, pudiesen ser indicativos de la persistencia de viejos y nuevos sexismos; y demandar - pues exigir no podemos -, un mayor compromiso de esta institución con modos de educación abiertamente antisexistas. Entendemos sería grave que en este nuevo milenio la lógica de la ciudadanía, suplantada por la de la mercantilización, y apoyada en la racionalidad técnica, olvidase que la igualdad de género es ingrediente fundamental para hacer efectivo el derecho a una ciudadanía plena que garantice sociedades del siglo XXI más democráticas, inclusivas y más justas.

12 Palabras clave: Educación universitaria. Ciudadanía global. Sexismos.

\section{Educação universitária e cidadania global: a igualdade de gênero pode ser optativa?}

\section{Resumo}

A partir das contribuições dos estudos sobre questões femininas, feministas ou de gênero, enfocaremos o contexto universitário com dois objetivos: evidenciar alguns dados que possam ser indicativos da persistência de velhos e novos sexismos; e demandar - pois exigir não podemos -, um maior compromisso desta instituição em desenvolver uma educação abertamente antisexista. Entendemos que seria grave neste novo milênio a lógica da cidadania, que tem sido suplantada pela da mercantilização, e apoiada na racionalidade técnica, esquecesse que a igualdade de gênero é ingrediente fundamental para tornar efetivo o direito a uma cidadania plena que garanta que as sociedades do século XXI sejam mais democráticas, inclusivas e mais justas.

Palavras-chave: Educação universitária. Cidadania global. Sexismos. 


\title{
University education and global citizenship: gendere quality can be optional?
}

\begin{abstract}
Based on the contributions of women's, feminist's or gender studies, this article focus on the university context with two purposes: to show some data that may be indicative of the persistence of old and new sexism; and demand - because we cannot require - a greater commitment of this institution to develop an education clearly against sexism. In this new millennium, when the logic of citizenship is being supplanted by mercantilism logic, and based on technical rationality, we understand that it would be serious if we forget that gender equality is a fundamental ingredient to materialize the right of a total citizenship and ensure that the society in the XXI century become more democratic, inclusive and fairer.
\end{abstract}

Keywords:University education. Global citizenship. Sexisms.

Nuestros campus están formados por ciudadanos y esto significa que debemos preguntarnos cómo debe ser un buen ciudadano de hoy y qué debe saber (NUSSBAUM, 2005).

¿Cómo debería transformarse la educación para la ciudadanía global en función de la consideración de los géneros, y especialmente del género femenino? (ARNOT, 2009).

\section{A modo de motivación}

En las sociedades definidas como democráticas hay cierta tendencia a dar por supuesta la igualdad - también la de género -, a considerarla una pieza definitivamente instalada en susdinámicas organizativas. Pero la realidad social, recogida a través de diversas investigaciones, informes y memorias, es tozuda, e insiste en demostrar que, lejos de ser un problema de épocas pasadas, las desigualdades entre mujeres y hombres están de rabiosa actualidad, lo que nos exige perfeccionar los análisis y seguir ejerciendo de "pepito grillo" - adoptando la metáfora de Celia Amorós (2005) -, paraavanzar en la construcción de sociedades más inclusivas y justas?'. 
Bajo esta motivación, nos preguntamos sobre el grado de persistencia/ superación de la segregación horizontal y vertical en educación, y buscamos identificar algunos de los factores que, enraizados en las culturas - como es el caso de los estereotipos de género -, pudiesen estar contribuyendo a la reproducción de formas de organización social de índole patriarcal. Queriendo partir del imaginario social, esto es, de lo que se podría denominar como cultura común en relación a esta temática, recurrimos a algunas de las voces del alumnado de $1^{\circ}$ curso del título universitario del Grado en Educación social de la Universidad de Coruña, aun siendo muy consciente de la debilidad en términos de representatividad.

Para finalizar, lejos de proponer conclusiones, recurrimos a la interpelación, esto es, a plantear nuevos interrogantes a viejos problemas: ¿̇Puede la formación universitaria asumir acríticamente los sexismos inseridos en sus dinámicas? ¿Reclama los estudios de género en el análisis de sus datos, las discriminaciones indirectas, los techos de cristal, los mecanismos de reconocimiento académico, la planificación de la carrera? ¿Se plantea explícitamente contribuir a la construcción de una ciudadanía sin marca de género? ¿Está dispuesta a que el argumentario feminista tenga carácter obligatorio?

\section{Algunos datos evidentes de segregación horizontal y vertical en educación}

Sin datos no hay visibilidad, sin visibilidad no hay credibilidad y sin credibilidad no hay prioridad (CASTAÑO, 2008).

La preocupación por la incorporación de la perspectiva de género a las estadísticas, por la necesidad de recoger, recompilar y presentar los datos analizados por sexoes una prácticacon apenas historia, esto es, relativamente reciente ${ }^{2}$. nel contexto español, la Ley orgánica 3/7, de 22 de marzo de 2007, para la igualdad efectiva de mujeres y hombres, introduce en su artículo 20 normas específicas sobre la incorporación del enfoque de género en las estadísticas, lo que exige incluir sistemáticamente la variable sexo yelaborar nuevos indicadores que mejoren el conocimiento de la realidad y las potenciales desigualdades entre mujeres y hombres. 
Disponer de estos indicadores, lejos de ser una cuestión trivial - de moda o pasajera -, es elemento imprescindible para el diseño de políticas de igualdad, pues como bien explica Cecilia Castaño (2008, p. 56) ampliando la afirmación de Nancy Hafkin "[...] sin datos no hay visibilidad, sin visibilidad no hay credibilidad y sin credibilidad no hay prioridad" 3 .

En este apartado acudimos a fuentes estadísticaspublicadas por el Ministerio de Educación, Ciencia y Deporte (ESPAÑA, 2014; 2015 ) para evidenciar segregación vertical y horizontal en educación. Empezamos recogiendo algunos datos para visibilizar el equilibrio versus desequilibrio en el acceso y en los logros del alumnado, así como en su distribución en las diferentes etapas y ramas científicas, y nos preguntamos si el éxito académico lleva aparejado el correspondiente éxito social con independencia de género ${ }^{4}$.

Adoptamos la conceptualización recogida en el Informe Eurydice $(2011)^{5}$, sobre segregación horizontal y segregación vertical respecto de las desigualdades de género en la educación superior, que hacemos extensible al resto del sistema educativo. Así, entendemos que la segregación horizontal se manifiesta cuando hombres y mujeres eligen diferentes ámbitos de estudio en la educación, y las mujeres están infrarrepresentadas en los de ingeniería y ciencias; mientras que en la segregación vertical el problema estaría relacionado con el "techo de cristal", es decir, que aunque el número de mujeres graduadas en educación superior supere al de hombres, en los estudios de doctorado están ligeramente infrarrepresentadas y su número es incluso menor entre el personal académico de las universidades ${ }^{6}$.

\subsection{Sexualización en la orientación profesional}

En la formación profesional, la división sexual en los ciclos formativos se mantiene de forma clara. Empezamos seleccionando los datos de alumnado matriculado en el Grado Medio, donde los porcentajes más destacadas de presencia de mujeres son: Imagen Personal (94,2\%), Servicios Socioculturales y a la Comunidad (85,6\%), Textil Confección y Piel $(86,9 \%)$ y Sanidad $(75,2 \%)$, mientras que están prácticamente ausentes de Mantenimiento y Servicios a la Producción (2\%), Mantenimiento de Vehículos Autopropulsados (2, 1\%), Fabricación Mecánica (3,4\%), Electricidad y Electrónica (4\%), Madera y Mueble $(4,9 \%)$ o Actividades Marítimo-Pesqueras (6,6\%). 
Porcentajes similares a los obtenidos en los Programas de cualificación profesional inicial, que nos da pistas sobre como la variable clase social se cruza con la de género, reforzando una elección fuertemente sexualizada, tal y como ilustra el siguiente gráfico?

\section{D4. LOS PROGRAMAS DE CUALIFICACIÓN PROFESIONAL INICIAL}

D4.9. Distribución porcentual del alumnado según sexo, por familia profesional

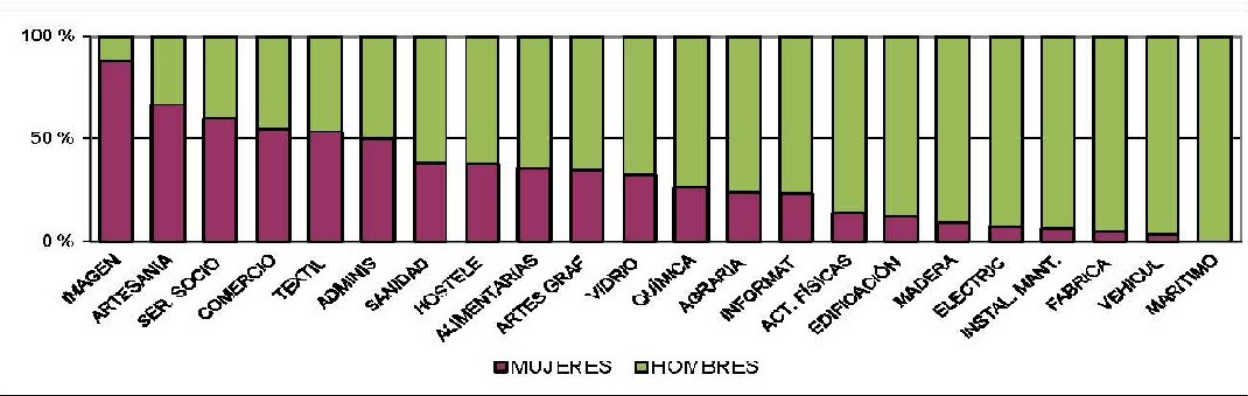

AGRARIA
MARITIMO
ALIMENTARIAS
OUIMICA
IMAGEN
SANIDAD
FABRICA
INSTAL. MANT.
ELECTRIC:
VLI IICUL
EDIHCACÓN

Acrari=
Maríimo-Pesquera
Industrias Alimentarias
Ouimica
Imagen Personal
Sanidad
Fabricación Mecánica
Ins:alación y Mantenimiento
Electricidad y Electrórica
Transp y IMantcnimicnto de Vch.
Edificasión y Obra Civil

\begin{tabular}{|c|c|}
\hline VIRRIO & Vidioy Cerámica \\
\hline NAT.FRA & Nadera, Mueble y Corcho \\
\hline TEXTIL & Textil, Confección y Piel \\
\hline ARTES GRAF & Artes Gráfices \\
\hline INFORMAT & Infcrmática y Comunicaciones \\
\hline ADMINIS & Administración y Gestión \\
\hline COMERCIO & Comerciny Wa keting \\
\hline SER SOCIO & Serv. Socioculturales y a la Cdad. \\
\hline HOS'ELE & Hostelería y Turismo \\
\hline ACT. IÍSICAS & Act vidades 「ísicas y Deportivas \\
\hline ARTESANIA & Autesanías \\
\hline
\end{tabular}

En el nivel de Bachillerato, la distribución porcentual del alumnado según modalidad cursada es la siguiente: En la rama de Humanidades y Ciencias Sociales, ellas un 56,1\% frente al 45,6\% de ellos; en el de Artes, un $5,9 \%$ frente al $4,0 \%$ de ellos y su presencia es menor únicamente en la de Ciencias y Tecnología, 38\% frente al 50,4\%. 


\section{Distribución porcentual del alumnado de Bachillerato según modalidad cursada}

(evolución y situación por sexo)

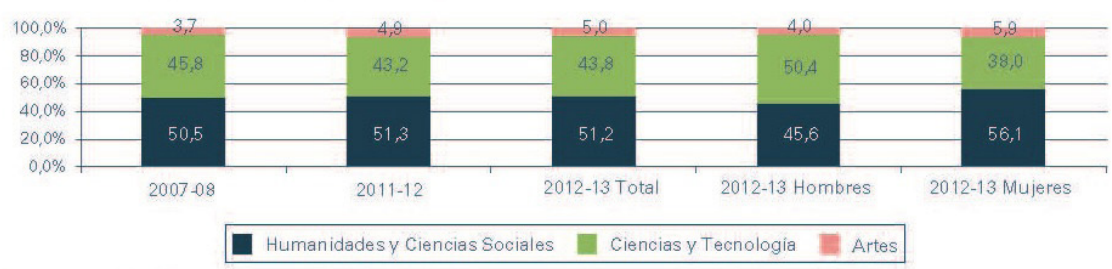

En el mismo curso académico, la presencia de mujeres matriculadas en Grado y $1^{\circ}$ y $2^{\circ}$ ciclo, es superior al de los varones, situándose en un $54.3 \%$ del total del alumnado matriculado, es además mayoritaria en todas las ramas de enseñanza universitaria, con la excepción de las titulaciones técnicas len Ciencias de la Salud el 70, 1\% son mujeres, en Artes y Humanidades el $61,6 \%$ en Ciencias Sociales y Jurídicas o 60,9\%, y en Ciencias o 52,6\%). Sin embargo, en la rama de Ingeniería y Arquitectura el porcentaje de mujeres se sitúa en el 26, $1 \%$.

Distribución similar ocurre en los estudios de Master donde las mujeres representan el 54, 1\% del total, distribuyéndose como sigue en las diferentes ramas: Ciencias de la Salud (69,1\%), Artes y Humanidades (62\%), Ciencias Sociales y Jurídicas $(56,8 \%)$, Ciencias $(50,4 \%)$ y subiría ligeramente en Ingeniería y Arquitectura (38\%). Segregación horizontal que también se destaca en el ya citado Informe de Eurydice.

Son también mayoría las estudiantes universitarias españolas en el programa Erasmus, representando el 55,4\% y alcanzando el 73,35\% en el doctorado.

Seleccionamos tres gráficos que, por su fuerza visual, intuimos que contribuyen significativamente al objetivo propuesto. 
Estudiantes matriculados y egresados en el Sistema Universitario por sexo.

\begin{tabular}{|c|c|c|c|c|}
\hline & \multicolumn{2}{|c|}{ Matriculados } & \multicolumn{2}{|c|}{ Eggresados $^{(2)}$} \\
\hline & Total & $\%$ de mujeres & Total & $\%$ de mujeres \\
\hline Total estudiantes & 1.561 .123 & $54,3 \%$ & 256.493 & $57,6 \%$ \\
\hline Estudiantes de Grado & 1046.570 & $55,5 \%$ & 31.072 & $57,2 \%$ \\
\hline Estudiantes de $1^{e t}$ y $2^{\circ}$ ciclo & $4 \hat{3} .466$ & $51,2 \%$ & 175.013 & $57,2 \%$ \\
\hline Estudiantes de Máster Oficial & 111. 587 & $54,1 \%$ & 59.803 & $56,6 \%$ \\
\hline
\end{tabular}

Distribución de los estudiantes matriculados en Grado y $1^{\text {er }}$ y $2^{\circ}$ Ciclo por rama de enseñanza y sexo 口Hombres aMujeres

Total

\begin{tabular}{|l|l|}
\hline $45,7 \%$ & $54,3 \%$ \\
\hline
\end{tabular}

Ciencias Sociales y Jurídicas

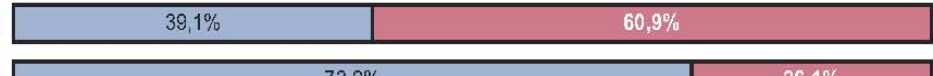

Ingeniería y Arquitectura Artes y Humanidades

Ciencias de la Salud

$$
\square
$$
Ciencias

\begin{tabular}{|c|c|}
\hline \multicolumn{3}{|c|}{$73,9 \%$} & $26,1 \%$ \\
\hline $38,4 \%$ & $61,6 \%$ \\
\hline $29,9 \%$ & $70,1 \%$ \\
\hline \multicolumn{2}{|c|}{$|c|$} \\
\hline \multicolumn{2}{|c|}{$57,4 \%$} \\
\hline
\end{tabular}

Distribución de los estudiantes matriculados en Máster por rama de enseñanza y sexo.

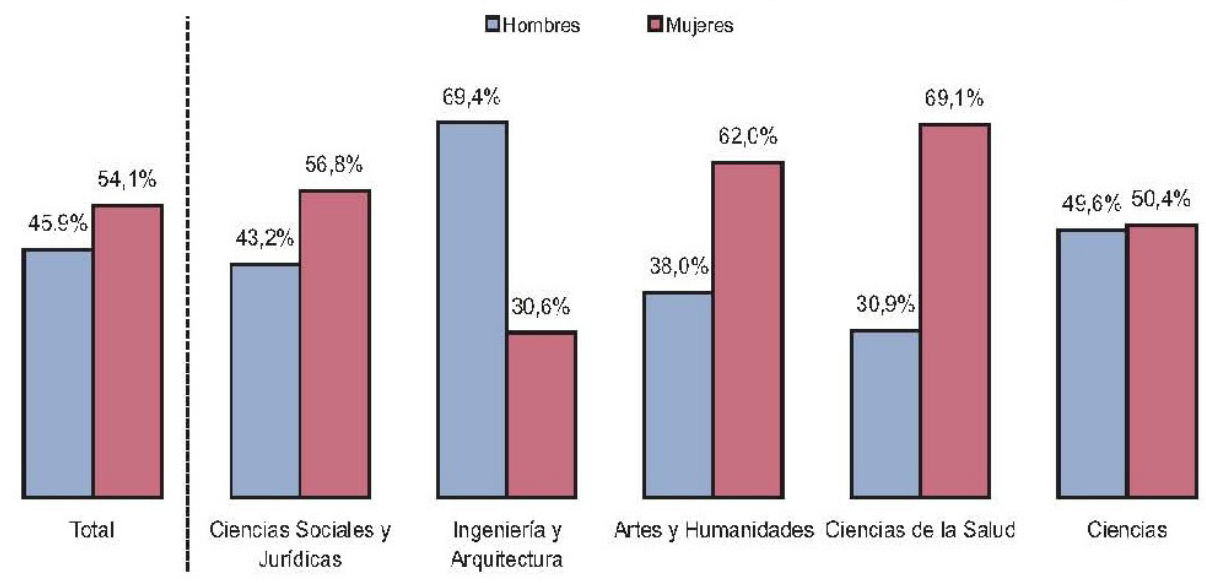




\subsection{Cuando el buen resultado académico no garantiza mejor ubicación social $^{8}$}

Las chicas tienen mejor rendimiento escolar en todas las etapas educativas: sacan mejores notas, abandonan menos, se esfuerzan y estudian más y por más tiempo; es decir, hay más chicos que chicas que repiten, que no acaban la secundaria, que no se gradúan. No sólo los resultados finales, sino toda la estancia en el sistema educativo parece ser mejor para las chicas que para los chicos. Más chicos que chicas van a la escuela porque los obligan. A ellas les gusta más estudiar, viven la trayectoria escolar ligada a la promoción personal, profesional y cultural; la aceptan como garantía de éxito personal que rompe definitivamente con el rol tradicional y prepara el camino para una autonomía personal y económica en la vida adulta.

Veamos a modo de ejemplo algunos porcentajes:

En bachillerato, las mujeres, además del legaren mayor proporción $(52,4 \%)$, obtien en mejores resultados que los hombres respecto a la promoción, sacándo les hasta casi cinco puntos de diferencia por encima de los chicos.

Superan la prueba de acceso a la universidad un $55,3 \%$ y el $54,1 \%$ del total de estudiantes universitarias son mujeres, incrementándosesu proporción entre los egresados hasta situarse en el 59,7\%.

En el ámbito universitario, directamente ligado con los créditos matriculados, presentados y aprobados se encuentran las tasas de rendimiento (relación entre créditos superados y créditos matriculados), éxito (créditos superados sobre presentados) y evaluación (créditos presentados sobre matriculados).

En las titulaciones de grado la tasa de rendimiento se sitúa en el $72,1 \%$, la de éxito en el 84,5\% y la de evaluación en el 85,2\%. En términos globales, la nota media del expediente académico en Grado, es de 7,04 frente al 6,83 de los varones y la de egresados en Master es de 8,21\% frente al $8,09 \%$ de hombres.

Este incremento en la presencia de las estudiantes en el espacio universitario, y su mejor rendimiento académico, suponen un gran avance en el reto de la igualdad, pero agudizando la mirada detectamos nuevas diferencias sobre las que es necesario sospechar y que desmienten el mito referido al carácter neutral de la educación. Parece que ahora que consiguieron el éxito 
Educación universitaria y ciudadanía global: ¿̨puede la igualdad de género ser optativa?

académico, la discriminación persiste en la valoración social y profesional del mismo.

Así, la tasa de paro de las mujeres con educación superior es ligeramente superior a los hombres: 16,7\% frente al 13,6\%. El porcentaje de egresados universitarios que están de alta en la seguridad social es ligeramente inferior en las mujeres: 47,5\% frente al $49 \%$ de los varones. Adjuntamos la tabla correspondiente al índice de salarios según el nivel de estudios.

\section{Índices de salarios por hora, según nivel de formación alcanzado.}

\begin{tabular}{lrrr|rrr}
\cline { 2 - 7 } & \multicolumn{3}{c|}{ Total asalariados } & \multicolumn{3}{c}{ Asalariados de 25-34 años } \\
\cline { 2 - 7 } & \multicolumn{1}{c}{ Total } & Hombres & Mujeres & Total & Hombres & Mujeres \\
\cline { 2 - 7 } TOTAL & 100,0 & 105,7 & $\$ 3,5$ & 100,0 & 104,3 & 95,4 \\
Educación Primaria o inferior & 74,6 & 79,6 & 66,1 & 75,3 & 80,1 & 65,1 \\
Primera etapa de E. Secundaria & 79,2 & $85, \mathrm{C}$ & 68,7 & 84,7 & 90,1 & 76,0 \\
Segunda etapa de E. Secundaria & 88,6 & 99,2 & 76,8 & 89,7 & 100,1 & 76,8 \\
Educación Superior & 123,8 & 131,7 & 116,6 & $11<, 3$ & 119,1 & 110,5
\end{tabular}

Fuente: Encuesta de Condiciones de Vida. IVE.

En cuanto a la distribución del profesorado de EE. De Régimen General no universitarias por sexo, la docencia es claramente una profesión femenina, pues las mujeres representan el 71, 1\%, estando sobrerrepresentadas en el nivel de Maestras (79,5\%), menos en el grupo de Catedráticas y Profes. E. Secundaria $(58,5 \%)$ y aún menor en el cuerpo de Prof. y técnicos de FP $(42,3 \%)$.

Sin embargo, en el colectivo de profesorado docente e investigador universitario, las profesoras son minoría, representando el 39,3\%, y su presencia es todavía menor en las figuras de catedrática $(20,3 \%)$, y emérita $(25 \%)$ y CEU (29,2\%).

También son menos en la categoría de Profesorado doctor/a, representando un $37,9 \%$.

En el cuerpo docente universitario con sexenios por sexo y rama, el porcentaje es superior en los varones, salvo en la rama donde la presencia de las mujeres es muy inferior: ingeniería y arquitectura, donde ellas destacan con 2,4 puntos porcentuales por encima. 
Los gráficos, una vez más, son iluminadores, diría incluso cegadores.

Distribución del PDI por sexo y categoría laboral .

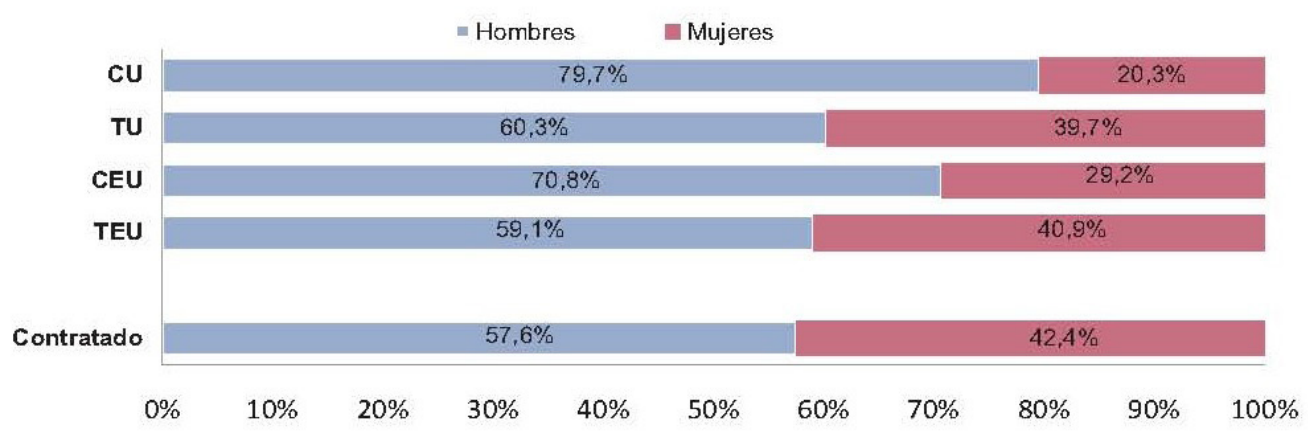

Distribución del profesorado funcionario por sexo.
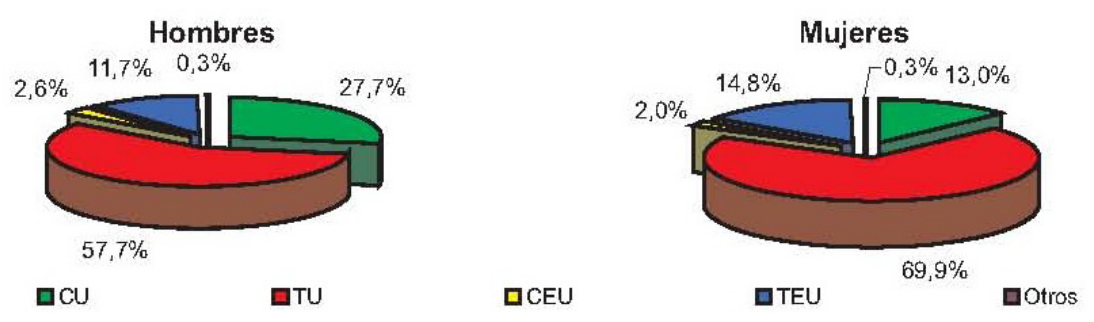

PDI Doctor por tipo de universidad y sexo.
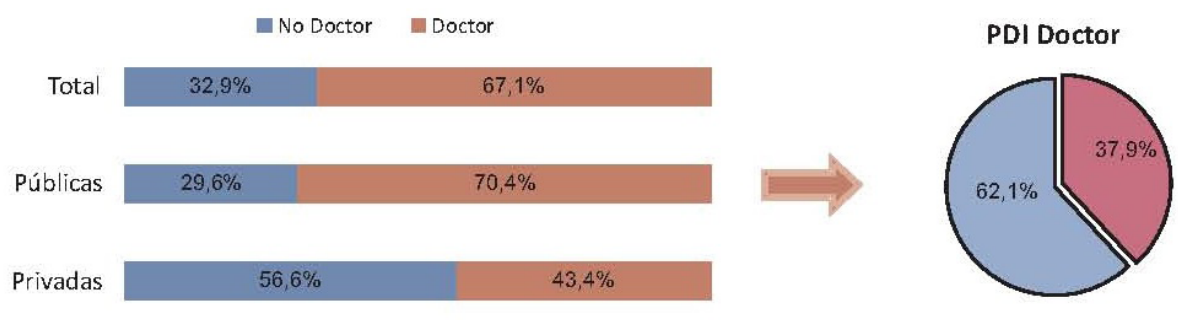

DMujeres DHombres 
Educación universitaria y ciudadanía global: ¿̇puede la igualdad de género ser optativa?

\section{Cuerpo Docente Universitario con sexenios por sexo y rama de conocimiento.}

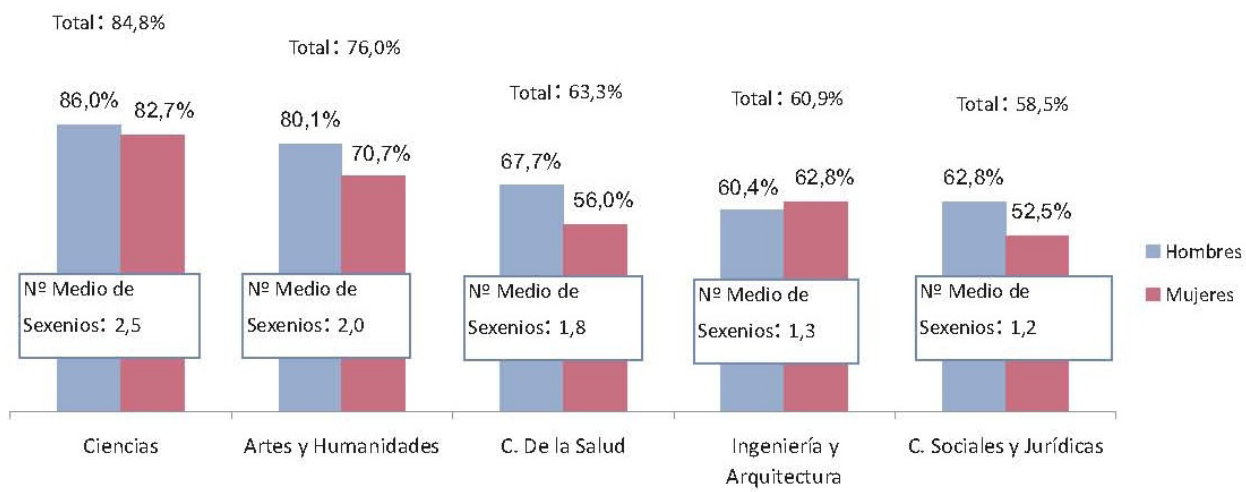

Sobre algunos de los mecanismos de autorización para el saber y sus ritos, explica brillantemente Amelia Valcárcel (2009, p. 125): "Se les ha concedido el estar, pero probablemente no el ser; de que estén con los sabios no se sigue que sean sabias. Eso sí, suplirán con aplicado y constante trabajo la capacidad de innovación que se les niega."

Distribución por sexo del investigador principal en el PN de Proyectos de Investigación Fundamental por tipo de centro

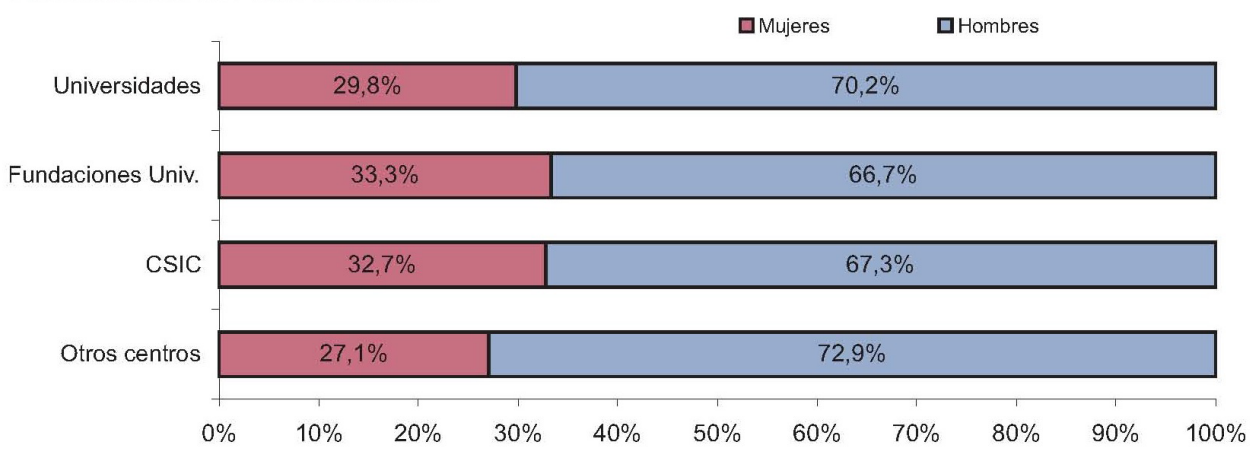

Resulta obvio, como manifiesta de forma tajante Raquel Osborne (2008, p. 102) "[...] que existe un déficit cuantitativo en lo relativo al número de mujeres que participa en los órganos de poder político y administrativo o en las más altas jerarquías del mundo del trabajo y de la empresa." Se constatan así algunos de los obstáculos derivados de la ausencia de masa crítica suficiente por parte de las mujeres, entendiendo por ésta no sólo un incremento 
en la cantidad relativa de mujeres, sino un cambio cualitativo en las relaciones de poder que le permite por primera vez a la minoría utilizar los recursos de la organización o de la institución para mejorar su propia situación y la del grupo al que pertenece" (VALCÁRCEL, 1997, p. 176). De no ser así, es fácil entender las razones del desinterés.

En el intento de explicar los motivos que subyacen al hecho de que el éxito académico no se traduce en situaciones más ventajosas para las mujeres en el mercado laboral, acudimos a la reflexión de Terry Wrigley (2007, p. 27), "[...] la investigación de la eficacia escolar (IEE) deriva cara a los resultados susceptibles de ser medidos, y especialmente a las puntuaciones de los exámenes", ningun eándose así, en nombre de la objetividad yla eficacia, otros resultados que también son producidos por la escolarización y que no se ajustan a eses moldes de medida.Por otro, porque las orientaciones profesionales de las chicas, como se suele asumir, sin mayor reflexión, se canalizan hacia profesiones feminizadas.

Las estadísticas muestran mejor rendimiento académico y por tanto mayores tasas de éxito en el sistema educativo por parte de las chicas, pero también muestran que las mujeres con una formación y titulación "no tradicional" tienen más dificultades para encontrar empleo.

Comprender esta realidad en sus dimensiones más profundas requiere, siguiendo a Nicole Mosconi ${ }^{10}$, acudir a múltiples explicaciones subjetivas, entre las que apunta que las chicas en sus decisiones operan con elecciones racionales contrarias al estereotipo tradicional perotienen en cuenta de manera realista las posibilidades efectivas de empleo que el mercado laboral les ofrece.

Así, otro de los aspectos de análisis es la propia concepción de "éxito" que comúnmente se maneja, como ya tenemos explicado en otro trabajo ${ }^{11}$. Para ser buen alumno o alumna, como explica el sociólogo Perrenoud (1990), no llega sólo con dominar el currículo, sino que requiere también, e quizás más, comprometerse en las actividades propuestas o impuestas y respetar las reglas. Esto es, para alcanzar el estatus de excelencia escolar resulta imprescindible cierto grado de conformidad. Conformidad con las convenciones gráficas, lingüísticas o matemáticas; pero conformidad también con las normas morales, en relación con los otros y con las tareas propuestas. Siguiendo la argumentación del brillante sociólogo, si un buen trabajo escolar se caracteriza - entre otros asuntos -, por no ser retribuido, en buena medida impuesto, fragmentado, 
repetitivo y supervisado constantemente, sin duda las chicas saben mucho de esto, ya que estos requerimientos ilustran a la perfección las competencias del trabajo doméstico y de cuidados, desarrolladas fundamentalmente por las mujeres a lo largo de la historia. He ahí una de las (e)videncias del éxito escolar más desatendida: "¡Tener éxito en la escuela supone aprender las reglas de juego!"12

El mayor "éxito" académico de las chicas, o tal como queda formulado su presunto control de las reglas de juego ¿̇stá significando para ellas mayores privilegios? ¿Qué papel tienen los estereotipos de género en la elección de estudios, en las preferencias, en los resultados académicos? ¿Qué responsabilidad tienen las instituciones educativas en las expectativas y opciones individuales?

Se podría pensar que esta dinámica consistente en otorgar privilegios al colectivo masculino y, por tanto, con efectos claramente discriminatorios sobre las mujeres, ya no es responsabilidad del sistema educativo, pues funciona fuera de su ámbito competencial; pero sus efectos en el reconocimiento, en las relaciones, en la configuración de las identidades, esto es, en posibilitar aprendizajes para ejercer una ciudadanía con o sin marca de género, son

24 conocimientos no asignaturizados que perduran más allá de las etapas escolares y de la superación o no de exámenes y pruebas. Se encuentran en las estructuras, en las raíces de organización social. Es allí a donde nos dirigimos en el siguiente apartado.

\section{Estereotipos de género y "éxito" social, o lo que no explican los datos}

"Detrás de comportamientos que consideramos normales, subyace una estructura de poder que acaba condicionando las formas de estar en el mundo de las personas según sean hombres o mujeres" (SUBIRATS; TOMÉ, 2007).

Ofrecer las mismas titulaciones, las mismas materias, no es suficiente para evitar los efectos de las socializaciones diferenciadas y, por consiguiente, la discriminación en relación con las aspiraciones. Incluso obteniendo mayores logros educativos que en el pasado - esto sí es demostrable - parece que a 
ellas se les niegue el derecho a la excelencia, pues continúan aprendiendo a ocupar un lugar secundario en la sociedad: menos ingresos, víctimas de violencia de género, dificultad de acceso a cargos de responsabilidad, especialmente en el ámbito de la empresa privada, tratadas cómo cuotas cuando los ocupan, asumiendo el grueso de las tareas domésticas, con escasa rebeldía personal. Unas y otros no aprenden con independencia de sexo a ganar, a perder, a competir, a sentir.

Los gráficos seleccionados reflejan que la variable sexo-género sí importa, e indican que el problema actual no se sitúa en el rendimiento final que tienen las chicas en el sistema educativo, sino en la elección de los estudios, que colocan a las alumnas en una diferente posición social y profesional.

Ante esta evidencia, y siguiendo la propuesta de la profesora Carmen Rodríguez, consideramos necesario analizar en profundidad en qué se sustentan tales preferencias, tales vocaciones o gustos, pues sabemos que lejos de remitir a argumentos de naturaleza, tienen un fuerte componente sociocultural. En palabras de la profesora

Las alumnas obtienen mejores resultados en aquello que estudian, pero siguen recibiendo un mensaje implícito que les indica su posición subordinada en los ámbitos sociales, profesionales y familiares, contribuyendo a la reproducción de la sociedad patriarcal [...]. La posibilidad de elección que brinda la sociedad democrática convierte en invisible la socialización diferenciada que reciben chicas y chicos (RODRÍGUEZ, 201 1, p. 74).

Sobre la distribución académica de las chicas en la universidad, Pilar Ballarín (2008) destaca dos asuntos. Por un lado, su canalización cara a opciones o carreras profesionales menos selectivas y de menor valoración en el mercado, sigue corroborando que los mejores resultados no se corresponden con una incorporación al mercado laboral más ventajosa; por otra, que la creciente feminización en determinadas ramas profesionales es indicativo de que las "chicas" se orientan cara a aquellas en que las mujeres ya tienen presencia posiblemente para evitar el doble esfuerzo que significa la incorporación laboral posterior a un espacio nuevo para las mujeres.

En el mismo sentido, pero concretando la reflexión alrededor de la llamada segunda brecha digital, Sainz y González (2008), coinciden en identificar los roles de género y estereotipos acerca de lo qué es la tecnología, y 
Educación universitaria y ciudadanía global: ¿̨puede la igualdad de género ser optativa?

quien tiene que estar en esta, como un fuerte obstáculo para su superación. Seleccionamos de su argumentario los siguientes puntos:

- A las mujeres se les atribuyen trazos expresivo-comunales, esto es, atributos relacionados con la dulzura, sociabilidad, emotividad y tendencia a expresar emociones. Por el contrario, a los hombres se les atribuyen características agéntico-instrumentales que hacen alusión a su capacidad para ser asertivos, agresivos, racionales y tendentes al logro (EAGLY; STEFFEN, 1984), creencias que contribuyen a perpetuar los estereotipos alrededor del papel de la mujer en la sociedad.

- Los estereotipos aglutinan una serie de expectativas procedentes de la observación de lo que hace la gente (la norma descriptiva) y de lo que se espera que la gente debería hacer (norma prescriptiva). En este sentido, cuanto menos encaje la imagen que el alumnado tiene de sí mismo con la imagen que tienen del típico estudiante matriculado en estos estudios, menor es la probabilidad de que escojan estas titulaciones.

- La amenaza del estereotipo tiene también una gran influencia sobre las actitudes que las mujeres muestran respecto a áreas consideradas tradicionalmente como masculinas. Las chicas anticipan las dificultades con que se encontrarán si escogen una carrera técnica, perciben que estos estudios requieren muchas horas de dedicación profesional y de renuncia a la vida personal, mientras que las carreras relacionadas con las Ciencias Sociales y las Humanidades son percibidas como más susceptibles de ser compatibles con la vida privada (SAINZ; LÓPEZ-SÁEZ; LISBONA, 2004).

- Las carreras que más demandan las chicas llevan consigo altas dosis de competencia (al tratarse de profesiones muy masificadas) y, por supuesto, llevan asociadas peores condiciones laborales (BRYNIN, 2006).

- Además, se tiene contrastado empíricamente el hecho de que los hombres que despuntan en un ámbito femenino obtienen más ventajas por esto; por el contrario, las mujeres que triunfan en ámbitos tradicionalmente masculinos se enfrentan a más dificultades y esfuerzos para alcanzar los mismos objetivos que sus compañeros, así como para demostrar que están igualmente capacitadas que ellos para ocupar el mismo puesto de trabajo.

Aunque los estudios sobre estereotipos de género señalan que los trazos de masculinidad y feminidad que se atribuyen a sí mismos hombres y 
mujeres van cambiando, no obstante, como destaca Esther Barberá (2006, p. 70), las creencias acerca de lo que los demás piensan siguen ancladas en estereotipos del pasado ${ }^{13}$. Podríamos pensar que nila evolución del autoconcepto, nilos cambios sociales que se produjeron ayudaron a cambiar los estereotipos. Pero no es así, los estereotipos cambian aunque tal vez nolo hagan en el sentido y con la velocidad que nos gustaría.

Un trabajo reciente de la profesora Marina Subirats nos presenta un buen ejemplo. A partir de historias de vida de mujeres nacidas en las décadas de los años setenta y ochenta estudiadas por Bárbara Biglia, Ángel Gordo y Pilar Parra ${ }^{14}$, muestra algunos cambios que se produjeron en las mujeres pero evidenciando también algunos hilos que nos conectan al pasado: "Las españolas de hoy ya no asumen mandatos: ceden a impulsos, indagan en sus deseos, corrigen sus opciones. Tantean, prueban, rectifican, aparentementeal azar. ¿2Pero qué eslo que, en el fondo, parece guiarlas, orientar sus búsquedas yactitudes?" 15

Pareciera que el núcleo del género femenino construido históricamente como "el ser para otro", sigue adherido a los procesos culturales de socialización, esto es, forma parte de la cotidianeidad, aunque sus versiones sean muy diversas. Tras las múltiples tareas asignadas a las mujeres se oculta la ausencia de identidad propia: "[...] un ser que debe ignorarse a sí mismo, que no es sino a través de otro (SUBIRATS, 2013, p. 774)." 16 Ynos hace observar como ese "ser para otro" se mantiene: "Subterráneo. Inconsciente. Desorientado. Deslocalizado. Por lo tanto, a menudo irreconocible" (SUBIRATS, 2013, p. $775)^{17}$.

Las mujeres ganaron en libertad pero la libertad conquistada, se sitúa en poder cambiar si así se quiere, pero "el otro" existe y resiste, no se atreve a "ser para sí":

Porque lo que cambió no es el mandato de ser para otro, para las mujeres, sinola concreción del otro. El 'otro', que en el pasado tenía una figura genérica, el hombre, y una concreción estricta, el marido, el hijo, el novio, el padre, el dios, el amo [...], es ahora una figura vacía, que ni siquiera tiene forma humana. Puede ser una causa, un movimiento social, una idea, una persona, una profesión o una construcción mental de cualquier tipo, siempre y cuando cumpla con la condición de presentarse como algo que trasciende a cada mujer (SUBIRATS, 2013, p.775)18. 
Pudiera ser relevante saber que piensa el alumnado sobre este asunto, cuáles son las creencias, valores y estereotipos implícitos en la vida cotidiana, esto es, que constituye la cultura que, en palabras de Celia Amorós "[...] no es más que el comportamiento común aprendido de la especie" (AMORÓS, 2005, p. 50).

¿Qué piensa la juventud sobre este asunto? ¿Da por conquistada la igualdad? ¿Cuál es el imaginario social, eso que la cultura asume como bien común?

\section{Un ejemplo de lo que piensa el alumnado sobre la (des)igualdad entre mujeres y hombres}

Adoptando el método de reflexión característico del enfoque crítico que, tal y como explica Wilfred Carr "[...] trata de educar a los profesionales promoviendo el conocimiento de sí mismos, con el fin de explorar la irracionalidad de sus creencias y prácticas de sentido común y localizar el origen de esta irracionalidad en el contexto institucionalizado y en las formas de vida social 28 de las que tienen surgido" (CARR, 2002, p. 75) 19; y asumiendo que en ocasiones los modelos críticos universitarios dejan poco espacio para evidenciar las contradicciones, las incertidumbres, la variabilidad contextual de las experiencias, entiendo que éstas pueden ampliar las teorías críticas y deben ser tenidas en cuenta para la construcción de nuevos saberes.

En la materia Género, Igualdad y Educación, ahora de carácter obligatorio en el recién estrenado título de Grado en Educación social, llevo quince años empezando cada curso de la misma manera: escuchando al alumnado. En la introducción a cada núcleo temático, busco sus opiniones que, no especialmente meditadas, arrojan luz sobre qué, cómo y dónde aprende la juventud a construir su imaginario con respecto a esta temática ${ }^{20}$.

Como tengo recogido en otros trabajos (IGLESIAS, 2011 ), las voces del alumnado, los argumentos que construyen para avalar lo que piensan, constituyen un buen ejemplode los supuestos de género arraigados en la ciudadanía, de los que selecciono los siguientes: creencia generalizada de que los sexismos actuales son residuales e incluso que se eliminarán a medida en que la sociedad avance; percepción de las relaciones personales como neutras en cuanto al género; rechazo e indignación ante los sexismos más 
vejatorios, pero cierto grado de complicidad compartida ante aquellos más sutiles, sobre los que el nivel de crítica es débil; desconocimiento de las contribuciones del feminismo social y académico; equiparación entre machismo y feminismo; tendencia a explicar las diferencias de género en términos que remiten al carácter natural, biológico; dificultades para percibir la necesidad de políticas de acción positiva ligadas al reconocimiento y a la redistribución; acercamiento al concepto de género, pero escasa referencia a la dimensión relacionada con el poder; opinión a priori sobre la mayoría de las temáticas, pero carencia de argumentación razonada y rigurosa, científica, sobre éstas; abunda la información procedente fundamentalmente de los medios, no resultado de una formación específica ${ }^{21}$.

Las voces recogidas iluminan cómo algunas de las raíces sexistas siguen prendiendo especialmente allí donde no parece necesario reflexionar: en lo dado por obvio, por dado, por compartido, por indiscutible que, como en el caso de los estereotipos, fuertemente instalados en el imaginario, tienen un peso fundamental en la representación simbólica de lo que es y de lo que debe ser una mujer y un hombre, como tenemos explicado en otro trabajo (SÁNCHEZ; IGLESIAS, 2008, p. 131 1). Además, son voces por veces incómodas, quizás porque el tema toca los modos de ser y de relacionarnos con el mundo. Toca la biografía personal: ique tiene tanto de sociocultural! En sus comentarios, podemos identificar, adoptando la metáfora de Celia Amorós (2005, p. 304) "[...] algunos de los hilos de color rosa tejidos en las redes educadoras, que son, las líneas y direcciones en las que podremos puntear el hilo violeta."

Coincidimos con Paul Gee $(2005)^{22}$, al considerar un imperativo moral hacer que estas opciones y supuestos convencionalizados o inconscientes sean menos consuetudinarios dado que suponen modelos culturales que, con cierta facilidad, puedan dañar a las personas. Por este motivo, es importante, como brillantemente propone el profesor Álvarez-Uría (2015), evidenciar como las instituciones sociales actúan coactivamente sobre sujetos y grupos sociales, operando a su vez como estructuras generativas de las conductas y mentalidades; esto es, se convierten en una especia de inconsciencia social que de forma mecánica, irreflexiva, guían la acción social de los sujetos en determinadas direcciones y, siguiendo al profesor, entendemos que para romper el desconocimiento, la amnesia, se hace imprescindible objetivar ese inconsciente social, esa dinámica oculta (ÁlVAREZ-URÍA, 2015). 
Así, tomando muy en serio este déficit democrático, sabiendo que tal desventaja no reside en las mujeres, sino en las relaciones sociales que mediatizan las oportunidades, resulta imprescindible identificar y desmontar las relaciones de poder que secularmente tanto tienen encorsetado los modos de vida de las personas, pues como explica $M^{a}$ Jesús Izquierdo:

La negación del poder comporta, que las relaciones en el recinto 'privatizado' no son relaciones entre iguales, sino relaciones de poder, y comporta, además, tener o poder de negar que las relaciones hombre mujer son relaciones sociales y no 'personales', si es que hay algo que se pueda definir como estrictamente persona ${ }^{23}$ (IZQUIERDO, 2006, p. 170).

En palabras de Rosa Cobo (2008, p. 40), "[...] la socialización de género, como las otras socializaciones, sólo puede ser combatida cuando se tiene conciencia de su existencia, es decir, cuando los individuos se dotan de marcos interpretativos da realidad social que desvelan las jerarquías no legíitmas, las dominaciones y las asimetrías sociales."

En el caso que nos ocupa, problematizar la ceguera de género, 30 requiere apelar a la formación, a la argumentación científica sobre esos asuntos, lo que exige a las universidades, para avanzar más y mejor, asumir el liderazgo crítico que le corresponde promoviendo propuestas feministas, que garantizará la innovación que requiere el contexto actual.

\section{La urgencia de incorporar las aportaciones de los estudios feministas a todas las titulaciones universitarias}

Las universidades incluirán y fomentarán en todos los ámbitos académicos la formación, docencia e investigación en igualdad de género y no discriminación de forma transversal (ESPANHA, 2004).

Coincidimos con la brillante filósofa Nussbaum (2005), al entender que la educación superior debe tener por meta crear una comunidad crítica, que busque la verdad más allá das barreras de clase, género y nacionalidad, que respete la diversidad y la humanidad de otros; y al proponer que cada universidad debería considerar el modo de incorporar estas cuestiones 
y perspectivas a cursos básicos obligatorios, de manera que el alumnado tenga la experiencia de ver a través del 'prisma de género', al considerar que produce una mejor ciudadanía, una ciudadanía más capaz de revisar temas complejos sobre las mujeres que, con seguridad, tendrán que manejar en sus vidas, tanto públicas como privadas; y que estas discusiones son valiosas para todo el estudiantado, no sólo para las mujeres.

Para que pueda cumplir con esta misión, la universidad debe garantizar que se recojan las contribuciones de los estudios feministas en las distintas disciplinas científicas, pues de no hacerlo, podría expedir títulos universitarios que reconocen tener superadas competencias para la práctica profesional sin formación ninguna sobre cuestiones que, como la relativa a la temática que nos ocupa, menguará la práctica de la igualdad, tanto en la vida privada como en la profesional y, por lo tanto, el desarrollo democrático de la sociedad en su conjunto.

El conocimiento científico no puede obviar los prejuicios que limitan la igualdad, no puede permitirse ser producido al margen del prisma de género. Los feminismos deben alcanzar todos los espacios universitarios, al entenderlo, siguiendo a Amelia Valcárcel como

[...] aquella tradición política de la modernidad, igualitaria y democrática, que mantiene que ningún individuo de la especie humana debe ser excluido de cualquier bien y de ningún derecho a causa de su sexo [...] Feminismo es pensar normativamente como si el sexo no existiese o no fuese relevante [...], pero se necesita porque si existe, si es normativo e además siempre es parcial y siempre de la misma manera: en contra de las oportunidades de las mujeres y sus derechos (VALCÁRCEL, 2009, p. 14).

En el contexto español, empezamos la primera década del siglo XXI con una serie de reformas legislativas que, al reconocer la existencia de discriminaciones en función de género, incidían en la necesidad de potenciar la igualdade entre mujeres y hombres en los ámbitos educativos, introduciendo diferentes medidas para eliminar las desigualdades en función de esta variable.

Así, el artículo 25 de la Ley Orgánica, del 22 de marzo de2007, exige que en el ámbito de la educación superior se promocione la inclusión en los planes de estudio de enseñanzas en materia de igualdad entre hombres y 
Educación universitaria y ciudadanía global: ¿̨puede la igualdad de género ser optativa?

mujeres, así como la creación de posgrados específicos y la realización de investigaciones sobre la materia.

Pero para el tema que nos ocupa, resulta especialmente significativa la Ley Orgánica, del 28 de diciembre del 2004, especificando en su exposición de motivos (aparto III), las obligaciones del sistema educativo para la transmisión de valores de respeto a la dignidad de las mujeres y a la igualdad entre hombres y mujeres y contemplar, para la Educación Secundaria, la incorporación de la educación sobre igualdad entre hombres y mujeres y contra la violencia de género como contenido curricular, incorporando en todos los Consejos Escolares un nuevo miembro que impulse medidas educativas a favor da igualdade e contra la violencia sobre a muller. Además, en su Título I, capítulo I, art. 4.7 establece: "Las Universidades incluirán y fomentarán en todos los ámbitos académicos la formación, docencia e investigación en igualdad de género y no discriminación de forma transversal".

De este breve resumen normativo se desprende que, entre otros retos, la Universidade debiera garantizar la inclusión de asignaturas troncales y obligatorias adaptadas a las diferentes titulaciones universitarias con el objeto de introducir las aportaciones de los estudios feministas en las distintas disciplinas,

32 teniendo en cuenta las que ya se imparten desde hace años como "asignaturas optativas" o de "libre elección"24.

$\dot{¿}^{E s}$ la transversalidad la solución? ¿Cuenta todo el profesorado con formación en estudios de género?

Los resultados de la Investigación Evaluación de la incidencia de los saberes de las mujeres, feministas y de género en la docencia universitaria ${ }^{25}$, quetenía como objetivo indagar en las creencias y prejuicios arraigados en el ámbito académico y que impiden el reconocimiento de los conocimientos construidos desde el feminismo, identifican algunos de los obstáculos que están a frenar la igualdad real, que podrían ser también algunos de los retos que tenemos pendientes. Sintetizamos los resultados sobre lo que piensa de la igualdad el profesorado universitario, recogidas por la profesora Ballarín Domingo (2011):

a) El desconocimiento de la contribución realizada por las mujeres a las diversas áreas de conocimiento lleva a considerar que el tratamiento de cuestiones de género en una disciplina depende del "interés personal" y lleva cada vez a 
más profesoras y profesores a tratar algunos temas por ser una cuestión social de moda y de corrección política.

b) Atender al análisis de género en los programas docentes es una cuestión de "sensibilidad" y "conciencia" - pero no de ciencia- por lo que explicar cuestiones relativas a las mujeres o al género no se considere que forme parte del TODO propio del programa (contenido que se considera científico, objetivo e impersonal, ya definido). Se puede añadir algún pequeño apéndice o matiz pero, en ningún caso, transformar lo existente.

c) La consideración de temática "social", hace que el profesorado universitario se ocupe de la temática en muchos casos por demanda del alumnado, en los trabajos, en algunas prácticas, o debates improvisados, siendo la "violencia de género" el tema de mayor interés desde todos los campos en que dicen ocuparse. Esta improvisación o forma incidental de hablar de cuestiones "concienciadoras", "de actitud", es lo que denomina el profesorado "transversalidad".

d) Al no reconocer, por no conocer, un saber específico, y pensar que lo que hay que saber sobre las mujeres es un conocimiento de tipo sociológico que procede de forma "natural" do nuestro contexto y nos "sensibiliza" y "conciencia" cara a la discriminación - cuestión de valores-, cualquiera puede "transversalizar" y se considera recomendable que así sea porque es una cuestión de "igualdad" - y "todos y todas lo somos ya" - y no del feminismo. Se entiende así que no se necesita ninguna formación específica para intervenir en la docencia sobre temas de igualdad y no discriminación. Sólo hace falta una "actitud" adecuada. En consecuencia se rechazan materias específicas.

e) Las creencias e prejuicios con respecto al feminismo no sólo persisten sino que parecen tenerse reforzado generando nuevas resistencias. El feminismo se considera radicalismo, algo del pasado. El término "feminista" adquirió muy diversos y antagónicos matices, desde ser utilizado como peyorativo por personas que afirman defender la igualdad, hasta ser asumido por otras sin más referentes que su oposición a la discriminación y a la violencia. La "igualdad", como algo ya conquistado, sirve de nuevo paraguas para acoger varones y mujeres, obviando agravios fruto de la idea de naturalezas distintas y complementarias que se siguen manteniendo.

f) La denominación de Género para referirse a estos Estudios, que emergen del feminismo, reconocida por las profesoras feministas como estratégicamente 
más oportuna al presentarse alejada de ese "halo" de ideologización de que se califica el feminismo, por una parte no acaba de librarse de los prejuicios que asocian "género" a "feminismo" y, por otra, genera confusión. Entre todos los malos usos y abusos del concepto hay que destacar entre los más nobles niveles de "asepsia" su creciente utilización como género gramatical.

g) Desde esa concepción da igualdad como un hecho, reaparece con fuerza el concepto de "persona" en que se disuelven y ocultan las diferencias de género. La desigualdad entre hombres y mujeres es una más entre las discriminaciones y en ellas se diluye.

h) Aquellas personas que no aceptanestas creencias pensando que "los otros" las tienen, contribuyen así a mantenerlas.

Las conclusiones expuestas por la profesora Ballarín no dan lugar a dudas y, coincidiendo con su argumentario, apelamos una vez más a sus palabras: "El éxito de los números no puede cegarnos. La promoción de la igualdad entre los sexos requiere algo más que un equilibrio numérico; exige un equilibrio conceptual y un esfuerzo consciente por corregir las desigualdades existentes que, por el momento, sigue pendiente" (BALLARíN, 2011 , p. 30).

Este equilibrio conceptual habrá que buscarlo en el paradigma feminista que, desde su nacimiento, contribuye a transformar las formas de pensar existentes, abriendo el camino a nuevas problematizaciones sociales e intelectuales (ÁLVAREZ-ÚRIA; VARELA, 2009, p. 39). Para reducir la brecha de este tipo de desigualdad, es necesario que profesionales desde los diferentes campos científicos impulsen acciones abiertamente antisexistas; profesionales que cuestionen las jerarquías sociales y practiquen modos de vida que contribuyan a globalizar un orden social comprometido en garantizar la igualdad entre mujeres y hombres y, por tanto, una sociedad del siglo XXI más democrática, inclusiva y más justa.

Para ir en esta onda, la formación universitaria debiera liderar un nuevo modelo de educación diseñado para una ciudadaníaglobal que, como brillantemente nos recuerda Madeleine Arnot," debe alentar a los futuros ciudadanos globales a que aprendan a 'indignarse': la ciudadana global es alguien que 'sabe cómo funciona el mundo, se indigna ante la injusticia y está, al mismo tiempo, dispuesto y capacitado para actuar, para enfrentarse a este desafío global" (OXFAM apud ARNOT, 2009) 
Las mujeres, como grupo, entraron en la universidad hace cien años. Desde aquella, y hasta ahora, se fue tejiendo una evolución indiscutible en la distribución equilibrada de varones y mujeres en esta institución. De nosotros depende, en gran parte, que la universidad del siglo XXI se caracterice por este compromiso: exigir la igualdad de género como ingrediente fundamental en el espacio universitario.

Para concluir, adoptamos las palabras de Rosalía Camacho:

No se trata únicamente de que logremos comer un trozo más grande de pastel lque existan más leyes 'a favor de la mujer', más mujeres abogadas, juezas, diputadas, más estudios que hablen del sector femenino de la población), sino realmente de lo que debemos ocuparnos para lograr verdaderos cambios es de cambiar la receta, o sea, de buscar e incluir otros ingredientes hasta ahora ausentes (la perspectiva de género, los métodos no sexistas de investigación), de manera que seamos parte de todos los trozos del pastel y no nos conformemos únicamente con una repartición más 'igualitaria' de un pastel con receta androcéntrica (CAMACHO, 1992, p. 6).

\section{Notas}

1 Estamos comprobando como en materia de igualdad también el retroceso, no sólo es posible, sino que puede avanzar muy rápido. El Grupo de Trabajo sobre la cuestión de la discriminación contra la mujer en la legislación y en la práctica del Consejo de Derechos Humanos dela ONU, que visitó por primera vez España entre el 9 y el 19 de diciembre de 2014, nos suspende en igualdad en todas y cada una de las áreas sobre las que la igualdad de género debe de asentarse para ser un hecho.

2 Tal y como recoge el Informe del Instituto de la Mujer (2009), este proceso se inicia en la Primera Conferencia Mundial sobre la Mujer en México(1975), al ponerse de manifiesto, por primera vez, que la falta de estadísticas y datos desagregados por sexo, así como la presentación de estos de modo apropiado para su uso por parte de agentes decisivos, es uno de los obstáculos más importante que impide cambiar la situación de las mujeres. Fue necesario esperar una década, hasta la tercera Conferencia Mundial sobre la Muller de Nairobi (1985), para que se tomasen algunas decisiones en este sentido a nivel mundial, al definirse 39 indicadores considerados como claves -entre ellos la educación-, para identificar la situación de las mujeres en el mundo. El impulso definitivo fue la Cuarta Conferencia Mundial sobre la Mujer en Beijing, hace 20 años. Incluso muchas personas sostienen que el verdadero nacimiento de las estadísticas con perspectiva de género, tal y como en la actualidad son concebidas, tiene aquí su punto de partida. Tiene dos implicaciones fundamentales: Por un lado, la asunción de un conjunto de objetivos que se recogen en la Plataforma de Acción de Beijing y que instan al desarrollo de las estadísticas de género y, por otro, la introducción del concepto de "transversalidad de la perspectiva de género". 
3 Cecilia Castaño Juan Martín e Susana Vázquez, "La brecha digital de género: acceso, uso y habilidades", en La segunda brecha digital, Dra. Cecilia Castaño (Valencia: Cátedra, 2008), 56.

4 Los datos recogidos para este apartado fueron tomados fundamentalmente de dos de las publicaciones más recientes del Ministerio de Educación, Cultura y Deporte. Los referidos a los niveles educativos no universitario de Ministerio de Educación, Cultura y Deporte, Datos y cifras. Curso escolar 2014/2015(Madrid: MECD, 2014a). http://www.mecd.gob.es/ servicios-al-ciudadano-mecd/dms/mecd/servicios-al-ciudadano-mecd/estadisticas/educacion/ indicadores-publicaciones-sintesis/datos-cifras/Datosycifras 1415.pdf. Los datos referidos a los estudios universitarios de Ministerio de Educación, Cultura y Deporte, Datos básicos del sistema universitario español. Curso 2013/2014 (Madrid: MECD, 2014b). http://www.mecd.gob. $\mathrm{es} / \mathrm{dms} / \mathrm{mecd} /$ educacion-mecd/areaseducacion/universidades/estadisticas-informes/datos cifras/DATOS_CIFRAS_13_14.pdf.

5 Informe de EURYDICE, Diferencias de género en los resultados educativos: medidas adoptadas y situación actual en Europa (Madrid, Secretaría de Estado de Educación y Formación Profesional, 201 1). http://eacea.ec.europa.eu/education/eurydice/documents/thematic_reports/120ES. pdf

$6 \quad$ Ibid., 97. El capítulo 8 se analizan las Políticas de igualdad de género en la educación superior.

7 Ministerio de Educación Ciencia y Deporte, Cifras de la educación en España. Curso 2012-2013 (Madrid: MECD, 2015). http://www.mecd.gob.es/servicios-al-ciudadano-mecd/estadisticas/ educacion/indicadores-publicaciones-sintesis/cifras-educacion-espana/2015.html.

8 Ma Elena Simón 2001; Nieves Blanco, 2001; Marina Subirats y Amparo Tomé, 2007; Marina Subirats, 2008; Pilar Ballarín, 2008; Iglesias, 2010; Iglesias y Ballarín, 2013.

369 Terry Wrigley, Escuelas para la esperanza. Una nueva agenda hacia la renovación (Madrid: Morata, 2007), 27.

10 Nicole Mosconi, Femmes et savoir. La société, l'école et la división sexuelle des savoires (París: EditionsL'Harmattan, 1994), $110-111$.

11 Ana Iglesias Galdo y Pilar Ballarín Domingo. "El mito del 'éxito' escolar de las chicas". En Revista Sarmiento, no 17 (Servizos de Publicacións das Universidades de Vigo, Coruña e Santiago de Compostela, 20131.

12 Philippe Perrenoud, La construcción del éxito y del fracaso escolar. Hacia un análisis del éxito, del fracaso y de las desigualdades como realidades construidas por el sistema escolar (Madrid: Morata, 1990), 223-226, 218.

13 Esther Barberá Heredia, "Aportaciones psicológicas al estudio de las relaciones de género", en Género y currículo. Aportaciones del género al estudio y práctica del currículo, comp. Carmen Rodríguez Martínez (Madrid: Akal, 2006), 70.

14 Pilar Parra Contreras: "El peso de la familia y del sistema educativo en las trayectorias de tres mujeres de clases populares, urbanas y rurales". Papers 98, n 4 (2013): 709-729; Ángel Juan Gordo López: "Los procesos de subjetivación de las nuevas élites culturales. Itinerarios de autonomía e (in)dependencia emocional de mujeres de clase media". Ibíd., 73 1-750; Barbara Biglia: "Experiencias fronterizas y efectos de las movilidades sociales y de clase". Ibíd., 75 1-772. Textos vinculados al proyecto de investigación dirigido por Julia Varela Fernández y financiado por la Secretaría de Estado de Universidades del Ministerio de Ciencia e Innovación (SEJ 2006-04 140).

15 Marina Subirats i Martori, "Mujeres y cambio social. En torno a los trabajos de Barbara Biglia, Ángel Gordo y Pilar Parra", Papers. Revista de Sociología. v. 98, n. 4, p. 773-777, 2013. 
16 lbid., 774.

17 lbid., 775 .

18 lbid., 775.

19 Wilfred Carr, Una teoría para la educación. Hacia una investigación educativa crítica, Madrid: Morata, 2002, p. 75.

20 La Facultad de Ciencias de la Educación de la UDC contemplaba en el plan de estudios de la Diplomatura de Educación social -desde su creación en el curso 1995-1996 hasta el 2.0092010- la materia Género y Educación, ofertada como optativa. En el 2009-2010 se estrena el título de Grado en Educación social, ofertándose con carácter obligatorio en primer curso la asignatura Género, Igualdad y Educación. Sobre las opiniones recogidas, aun no pretendiendo afirmar que todo el alumnado comparta aquellas seleccionadas como relevantes, creo que contribuyen a objetivar algunas de las raíces sexistas que siguen prendiendo especialmente allí donde no parece necesario reflexionar: en lo dado por obvio, por dado, por compartido, por indiscutible.

21 Esta elaboración personal sobre las opiniones recogidas a lo largo de los años como docente de esta materia, coinciden con los resultados de diversas investigaciones sobre el tema. Valgan como ejemplo: Ministerio de Sanidad, Servicios Sociales e lgualdad (2015) "Percepción social de la violencia de género en la adolescencia y la juventud".http://www.msssi.gob.es/ssi/ violenciaGenero/publicaciones/estudiosinvestigaciones/PDFS/Percepcion__Social__VG__ Adolesc_Juv.pdf. CENTRO REINA SOFÍA Y (2013): "Jóvenes y género, el estado de la cuestión "Informe del Centro Reina Sofía sobre Adolescencia y Juventud de la Fundación Ayuda con la Drogadicción (CRS/FAD).http://adolescenciayjuventud.org/es/publicaciones/monografias-y-estudios/item/jovenes-y-genero. EMAKUNDI (2014): "Estudio sobre la percepción del sexismo en la publicidad entre alumnado adolescente". Comunidad Autónoma de Euskadi.http://www. emakunde.euskadi.eus/contenidos/informacion/publicaciones_subvencionadas/es_def/adjuntos/beca.2013.3.estudio.percepcion.sexismo.pdf

22 James Paul Gee, La ideología de los discursos (Madrid: Morata, 2005), 101.

23 Ma Jesús Izquierdo, "As ordes da violencia: especie, sexo e xénero", en Políticas públicas de igualdade. Perspectiva de xénero, coord. Ana Sánchez Bello (Santiago: Laiovento, 2006), 170.

24 Como se recoge en la Declaración por la integración do estudio de las mujeres, feministas y de género en el espacio europeo de educación superior: "Sobre la evolución y solidez de este tipo de Estudios en las Universidades del Estado español en las últimas décadas dan buena cuenta la presencia de estos estudios en los curricula universitarios, a través de asignaturas optativas, de libre elección y a través de Programas de Doctorado".

25 "Evaluación de la incidencia de los saberes de las mujeres, feministas y de género en la docencia universitaria" Plan Nacional I+D+l Acción Estratégica sobre Fomento de lgualdad de Oportunidades entre mujeres y hombres 2007, N. '074/07".

\section{Referências}

ALVAREZ-URÍA, Fernando. El reconocimiento de la humanidad. Madrid: Morata, 2015. 
AMORÓS, Celia. Globalización y orden de género. En: AMORÓS, Celia e DE MIGUEL, Ana (Eds.). Teoría feminista: de la llustración a la globalización. De los debates sobre el género al multiculturalismo. Madrid: Minerva,2005.

ARNOT, Madeleine. Coeducando para una ciudadanía en igualdad. Madrid: Morata, 2009.

BALLARÍN DOMINGO, Pilar. Retos de la escuela democrática. En: COBO, Rosa (Ed.). Educar en la ciudadanía: perspectivas feministas. Madrid: Los libros de la catarata,2008.

Educación e políticas de igualdade. En: IGLESIA, Ana Galdo. Educando en igualdade. Coruña: Instituto de Estudios Políticos y Sociales, 2011.

BARBERÁ HEREDIA, Esther. Aportaciones psicológicas al estudio de las relaciones de género. En: RODRíGUEZ, Carmen (Ed.). Género y currículo. Aportaciones del género al estudio y práctica del currículo. Madrid: Akal, 2006.

BIGLIA, Barbara. "Experiencias fronterizas y efectos de las movilidades sociales y de clase". Papers. Revista de Sociología, Barcelona, v. 98, n. 4, p. 751-772, trimestral 2013

BRYNIN, Malcolm. Gender, technology and jobs. The British Journal of Sociology, v. 57, n. 3, p. 437-453, set. 2006.

CAMACHO, Rosalía. Presentación. En: FAClO, Alda. Cuando el género suena cambios trae. (Una metodología para el análisis de género del fenómeno legall), Costa Rica: ILANUD, 1992.

CARR, Wilfred. Una teoría para la educación. Hacia una investigación educativa crítica. 3. ed. Madrid: Morata.2002.

CASTAÑO, Cecilia. La segunda brecha digital. Valencia: Cátedra,2008.

COBO, Rosa. Repensando la democracia: mujeres y ciudadanía. En: COBO, Rosa (Ed.). Educar en la ciudadanía. Perspectivas feministas. Madrid: Catarata,2008.

El género en las ciencias sociales. Revista de educación. Educar monográfico coeducación. Jalisco: Secretaría de Educación, 2006.

GEE, James Paul. La ideología de los discursos. Madrid: Morata, 2005.

EAGLY, Alice H.; STEFFEN, Valerie. Gender stereotypes stem from the distribution of women and men into social roles. American Psychological Association, Washington (EUA), v. 46, n. 4, p. 735-754, 1984. 
EURYDICE, Informe de. Diferencias de género en los resultados educativos: medidas adoptadas y situación actual en Europa. Bruselas: Agencia Ejecutiva en el Ámbito Educativo, Audiovisual y Cultural, 2011.

GORDO, Ángel Juan. Los procesos de subjetivación de las nuevas élites culturales. Itinerarios de autonomía e (in)dependencia emocional de mujeres de clase media. Papers. Revista de Sociología, Barcelona, v.98, n. 4, p. $731-750$, trimestral 2013.

IGLESIAS, Ana.Xénero, igualdade e (co) educación social na UDC: da optatividade á necesaria obrigatoriedade. CEESG. Revista do Colexio de Educadoras e Educadores Sociais de Galicia, Lisboa, n. 9, p. 44-51, 2011.

IGLESIAS, Ana Galdo; BALLARÍN, Pilar Domingo. El mito del 'éxito' escolar de las chicas. Revista Sarmiento, Anuario Galego de Historia da Educación, Galicia, n. 17, p.67-82, 2013.

IZQUIERDO, Maria Jesús. As ordes da violencia: especie, sexo e xénero. En: BELLO, Ana Sánchez (Coord.) Políticas públicas de igualdade. Perspectiva de xénero. Ed. Laiovento, 2006.

ESPAÑA. Ley orgánica 1, del 28 de diciembre de 2004. medidas de protección integral contra la violencia de género. Boletín Oficial del Estado, Madrid, n. 313 , p. $42166-42197$, 29 dic. 2004.

Ley orgánica 3, del 22 de marzo de 2007.Para la igualdad efectiva de mujeres y hombres. Boletín Oficial del Estado, Madrid, n. 71, p. $42166-42197,23$ mar. 2007.

Ley Orgánica 4, del 12 de abril de 2007.Por la que se modifica la Ley Orgánica 6, de 21 de diciembre de 2001, de Universidades. Boletín Oficial del Estado, Madrid, n. 89, p. 16241-16260, 13 abr. 2007.

Ministerio de Educación, Cultura y Deporte. Las cifras de la educación en España. Curso 2012-2013 (Edición 2015). Madrid: MECD, 2015.

Ministerio de Educación, Cultura y Deporte. Datos y cifras curso escolar 2014/2015. Madrid: MECD, 2014.

- Ministerio de Educación, Cultura y Deporte. Datos básicos del sistema universitario español. Curso 2013/2014. Madrid: MECD, 2014a.

MOSCONI, Nicole. Femmes et savoir. La société, l'école et la división sexuelle des savoires.París: Editions L'Harmattan, 1994. 
NUSSBAUM, Martha Craven. El cultivo de la humanidad. Una defensa clásica de la reforma en la educación liberal. Barcelona: Paidós, 2005.

OSBORNE, Raquel. Mecanismos de creación y reproducción de la desigualdad. En: PULEO, Alicia (Ed.). El reto de la igualdad de género. Nuevas perspectivas en ética y Fiolosofía Política. Madrid: Biblioteca Nueva, 2008.

PARRA, Pilar. El peso de la familia y del sistema educativo en las trayectorias de tres mujeres de clases populares, urbanas y rurales. Papers. Revista de Sociología, Barcelona, v.98, n. 4, p. 709-729, trimestral, 2013.

PERRENOUD, Philippe. La construcción del éxito y del fracaso escolar: hacia un análisis del éxito, del fracaso y de las desigualdades como realidades construidas por el sistema escolar. Madrid: Morata. 1990.

RODRÍGUEZ, Carmen. Género y cultura escolar. Madrid: Morata, 2011.

SAINZ, Milagros; GONZÁlEZ, Ana. La segunda brecha digital: educación e investigación. En: CASTAÑO, Cecilia. La segunda brecha digital. Valencia: Cátedra, 2008.

SAINZ, Milagros; LÓPEZ-SÁEZ, Mercedes; LISBONA, Ana. Expectativas de rol profesional de mujeres estudiantes de carreras típicamente femeninas o masculinas, Acción Psicológica,

40 Madrid, v. 3, n. 2, p. 11-123, trimestral, 2004.

SÁNCHEZ, Ana; IGLESIAS, Ana. Curriculum oculto en el aula: estereotipos en acción. En: $\mathrm{COBO}$, Rosa. Educar en la ciudadanía: perspectivas feministas. Catarata, 2008.

SUBIRATS, Marina Martori. Mujeres y cambio social. En torno a los trabajos de Barbara Biglia, Ángel Gordo y Pilar Parra. Papers. Revista de Sociología, Barcelona, v. 98, n. 4, p. 773-777, trimestral 2013.

SUBIRATS, Marina; TOMÉ, Amparo. Balones fuera: reconstruir los espacios desde la coeducación. Barcelona: Octaedro, 2007.

SUBIRATS, Marina. La masculinidad hoy: un género obsoleto. En: W.AA. Actas del Congreso internacional e interdisciplinar Mundos de Mujeres Women's Worlds 2008: La igualdad no es una utopía. Nuevas fronteras: avances y desafíos. Universidad Complutense de Madrid, 2008.

VALCARCEL, Amelia. La política de las mujeres. Madrid: Cátedra, 1997.

Feminismo en el mundo global. Madrid: Cátedra, 2009. 
WRIGLEY, Terry. Escuelas para la esperanza: una nueva agenda hacia la renovación. Madrid: Morata, 2007.

Profa. Dra. Ana Iglesias Galdo Universidad de Coruña | Espanha Facultad de Ciencias de la Educación Grupo de Investigación Política Educativa, Historia e Sociedade (UDC) E-mail | ana.iglesias@udc.es

Recebido 3 ago. 2015 Aceito 11 abr. 2016 